\title{
Arachnoid cysts: case series and review of the literature
}

\author{
Gustavo Pradilla, M.D., ${ }^{1}$ ANd George Jallo, M.D. ${ }^{1,2}$ \\ Departments of ${ }^{I}$ Neurosurgery and ${ }^{2}$ Pediatrics, The Johns Hopkins School of Medicine, \\ Baltimore, Maryland
}

\begin{abstract}
Object. Arachnoid cysts are commonly encountered fluid collections in the central nervous system. Recent advances in neuroimaging have translated into an increased detection of these cysts; that is, diagnoses can be made more frequently at earlier stages. Significant advances have also been made in the surgical management of these lesions. The authors report on a case series that illustrates the diverse forms of presentation and the treatment modalities commonly used for arachnoid cysts.

Methods. Between January 2002 and December 2006, 20 patients with arachnoid cysts underwent surgery performed by the senior author at The Johns Hopkins Hospital. Seventy percent of the cysts were supratentorial, 5\% infratentorial, and 25\% spinal. All diagnoses were confirmed on neuroimaging, surgery, and histological examination. Included in the study were 12 male (60\%) and eight female (40\%) patients, ranging in age from 2 weeks to 39 years (mean age 10.9 years) at the time of surgery.

Symptoms at presentation included headache (41\%), weakness $(23 \%)$, seizure (14\%), hydrocephalus (9\%), scoliosis $(4 \%)$, cognitive decline (4\%), and visual loss (4\%). Twenty-five percent of the cysts were sylvian, $20 \%$ intraventricular, and $20 \%$ suprasellar. Supratentorial cysts were treated endoscopically in $73 \%$ of patients and with open resection in the remaining $27 \%$. Complications included spasticity, hemiparesis, cerebrospinal fluid leak, hydrocephalus, and subdural hygroma. On follow-up evaluation, $60 \%$ of patients had stable cysts and improved symptoms, $13 \%$ complete symptom resolution, and $13 \%$ stable symptoms.

All patients with spinal cysts underwent laminectomies and fenestration, and one cystosubarachnoid (CS) shunt was placed. Cyst reaccumulation occurred in three patients; two patients required cystoperitoneal (CP) and CS shunts. No deaths occurred.

Conclusions. Most arachnoid cysts are found incidentally and can be managed conservatively. Symptomatic patients are surgical candidates. Treatments include CP shunt placement, craniotomy, or endoscopic fenestration, and stereotactic aspiration. Recent advances in neurosurgical techniques and neuroendoscopy continue to favor fenestration over shunt insertion as the method of choice for initial cyst decompression.
\end{abstract}

\section{KEY WORDS • arachnoid cystd • surgery • craniotomy • outcome}

A RACHNOID CYSTS are congenital collections of CSF contained within the arachnoidal membrane and subarachnoid space $^{19}$ of the cisterns and major cerebral fissures. Although some cysts remain stable, others can expand and compress surrounding structures, prompting evaluation. ${ }^{3,19,24}$ Most of these lesions become symptomatic in early childhood; hence, 60 to $90 \%$ of all patients with arachnoid cysts are children., ${ }^{8,11,12}$

In 1831 Bright $^{4}$ first defined intracranial arachnoid cysts, describing them as "serous cysts forming in connection with the arachnoid and apparently lying between its layers." Overall, arachnoid cysts account for approximately $1 \%$ of all intracranial mass lesions. ${ }^{1}$ Data from autopsy studies have shown a low incidence ranging from 0.1 to $0.7 \%$, a rate that correlates with that in studies of cranial MR images, which show an incidence of $0.75 \%$. Most cysts are incidentally diagnosed during neuroimaging after head trauma. Prenatal ultrasonography as well as increased access to computed tomography and MR imaging has allowed cyst detection at earlier ages; hence, most cysts are recognized during the first two decades of life.

Abbreviations used in this paper: $\mathrm{CP}=$ cystoperitoneal; $\mathrm{CSF}=$ cerebrospinal fluid; $\mathrm{MR}=$ magnetic resonance.
The overall male/female predominance is approximately 2:1. Multiple or bilateral arachnoid cysts are unusual, and familial occurrence has been reported in only a few cases. ${ }^{9}$ An association between bilateral sylvian fissure arachnoid cysts and glutaric aciduria Type I has been demonstrated. ${ }^{14}$

Arachnoid cysts may result from either developmental defects or trauma. Congenital cysts appear to originate from alterations in CSF flow in the early phase of subarachnoid space formation. ${ }^{15}$ In some cases, these cysts are seen in patients with corpus callosum agenesis and malformations of the cerebral venous systems, which suggest a more pronounced developmental defect. Traumatic arachnoid cysts appear to occur following minor closedhead injuries in infancy and can result from arachnoid splitting within incomplete cisterns during infancy. Pathological examination usually reveals a cyst wall that resembles normal arachnoid; however, the cyst location seems to determine its pathological appearance. Whereas posterior fossa cysts display choroid plexus remnants, suprasellar and prepontine cysts exhibit neuroglial elements that resemble those found on the floor of the third ventricle.

Although most arachnoid cysts remain stable throughout life, some can enlarge due to various mechanisms such 
as active CSF secretion from the cyst membrane, ${ }^{19}$ osmotic gradients caused by a higher protein concentration in the cyst fluid, or cyst contents communicating with the subarachnoid space via ball-valve mechanisms that entrap CSF during Valsalva maneuvers. ${ }^{5,21,22}$ Cysts can rupture following minor trauma, resulting in subdural hygromas and intracranial hypertension, ${ }^{1}$ and can occur in association with traumatic subdural hematomas. ${ }^{16}$ Supratentorial cysts are more frequently encountered, with nearly half of all cysts occurring within the sylvian fissure. ${ }^{19}$ Less frequent locations include the interhemispheric fissure and the periclival region. Cysts of the suprasellar region are more commonly encountered in children than in adults. ${ }^{20}$

We retrospectively report on a consecutive series of patients with arachnoid cysts that were surgically treated by the senior author. Our data illustrate the common clinical features encountered, available surgical options, complications, and patient outcomes after surgical treatment of arachnoid cysts.

\section{Clinical Material and Methods}

Between January 2002 and December 2006, 20 patients with arachnoid cysts underwent surgery performed by the senior author at The Johns Hopkins Hospital. Fourteen of the cysts were supratentorial (70\%), one was infratentorial $(5 \%)$, and five were spinal (25\%). All diagnoses were confirmed on neuroimaging, surgery, and histological examination. Tables 1 to 4 summarize cyst location, preoperative clinical symptoms, complications, and outcome. The study included 12 male $(60 \%)$ and eight female (40\%) patients, ranging in age from 2 weeks to 39 years (mean age 10.9 years) at the time of surgery.

\section{Results}

Symptoms at presentation included headache $(41 \%)$, weakness (23\%), seizures (14\%), hydrocephalus (9\%), scoliosis (4\%), cognitive decline (4\%), and visual loss (4\%). No deaths occurred among patients in the study.

\section{Intracranial Cysts}

Fifteen patients presented with intracranial cysts: five in the sylvian fissure $(25 \%)$, four in the intraventricular region $(20 \%)$, four $(20 \%)$ in the suprasellar area, one in the temporal lobe, and one in the infratentorial region. Whereas endoscopic fenestration was performed in 11 patients (involving 73\% of the supratentorial cysts), a direct approach with marsupialization and fenestration of the cyst was used in only four patients (involving 27\% of the supratentorial cysts). All patients with suprasellar lesions

TABLE 1

Preoperative symptoms in 20 patients with arachnoid cysts

\begin{tabular}{ll}
\hline \multicolumn{1}{c}{ Symptom } & No. (\%) \\
\hline headache & $9(41)$ \\
weakness & $5(23)$ \\
seizure & $3(14)$ \\
visual loss & $1(4)$ \\
cognitive decline & $1(4)$ \\
hydrocephalus & $2(9)$ \\
scoliosis & $1(4)$ \\
\hline
\end{tabular}

TABLE 2

Locations of arachnoid cysts in 20 patients

\begin{tabular}{ll}
\hline \hline \multicolumn{1}{c}{ Location } & No. (\%) \\
\hline supratentorial & \\
suprasellar region & $4(20)$ \\
lat ventricle & $3(15)$ \\
3rd ventricle & $1(5)$ \\
sylvian fissure & $5(25)$ \\
temporal lobe & $1(5)$ \\
infratentorial & $1(5)$ \\
midline region & \\
spinal & $2(10)$ \\
cervical region & $2(10)$ \\
thoracic region & $1(5)$ \\
cervical-thoracic region &
\end{tabular}

underwent endoscopic fenestration. There were no postoperative complications directly related to the surgeries in nine patients $(60 \%)$ with intracranial cysts. Among the remaining patients, one had persistent spasticity, one suffered mild hemiparesis, one had a CSF leak, two had hydrocephalus (one was treated with a second successful fenestration and the other with ventriculoperitoneal shunt placement), and one patient had a subdural hygroma requiring bur hole drainage. On follow-up evaluation, two patients $(13.3 \%)$ demonstrated cyst reduction and complete symptom resolution, two patients $(13.3 \%)$ had decreased cyst sizes and improved symptoms, nine patients $(60 \%)$ had stable cyst sizes with improvement in their symptoms, one patient $(7 \%)$ had hydrocephalus due to cyst increase and required placement of a ventriculoperitoneal shunt, and one patient (7\%) was lost to follow up.

\section{Spinal Cysts}

Spinal arachnoid cysts were present in five patients: cervical and thoracic cysts in two patients each and a cervical-thoracic cyst in one patient. All of these patients underwent laminectomies and fenestration, and in one patient a cystosubarachnoid shunt was placed. Postoperatively, reaccumulation of the cyst occurred in three patients, two of whom required $\mathrm{CP}$ and cystosubarachnoid shunts and one of whom had mild postoperative hemiparesis that improved. On follow-up evaluation, one patient had a decreased cyst and improved symptoms, two had cysts that remained stable and controlled symptoms, one had cyst reaccumulation and stable symptoms, and one demonstrated cyst expansion that required shunting.

\section{Discussion}

Our data illustrate the diverse spectrum of presentations

TABLE 3

Treatments according to cyst location

\begin{tabular}{llr}
\hline \hline Cyst Location & \multicolumn{1}{c}{ Treatment } & No. \\
\hline supratentorial & craniotomy & 4 \\
& endoscopic fenestration & 11 \\
infratentorial & craniotomy & 1 \\
spinal & laminectomy & 5 \\
\hline
\end{tabular}



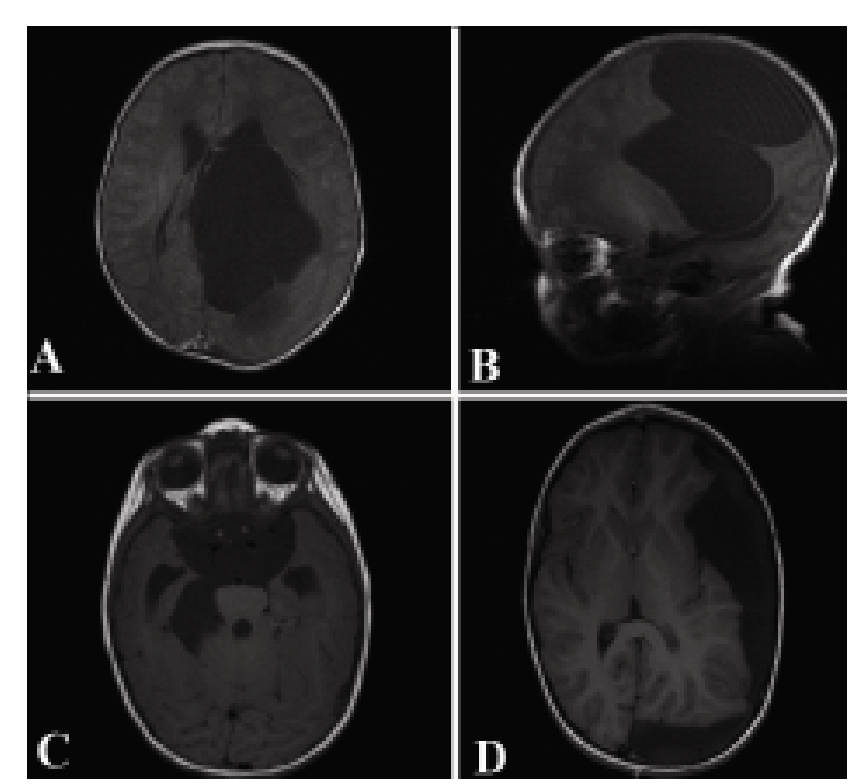

FIG. 1. Axial (A, C, and D) and sagittal (B) $T_{1}$-weighted MR images showing arachnoid cysts. Left porencephalic arachnoid cyst in a 2-week-old patient (A and B). Suprasellar arachnoid cyst in an 11-month-old patient (C). Left sylvian arachnoid cyst in a sixyear-old patient (D).

in patients with arachnoid cysts, available surgical options, and the complications and surgical outcomes in a selected population treated by a single surgeon. Although all patients underwent surgical treatment, not all arachnoid cysts require surgical intervention. ${ }^{5}$ On patient presentation, clinical decision-making is based on symptomatology and neuroimaging findings. Patients with asymptomatic or incidental cysts should be monitored clinically and with serial MR imaging. Surgical management should be considered only for increasing cysts causing neural compression, hydrocephalus, or refractory symptoms attributable to mass effect. Postoperative cyst reaccumulation has been reported to occur in approximately $25 \%$ of patients (Fig. 1). ${ }^{17,25}$

Surgical treatment options remain controversial and most commonly include either craniotomy, open fenestration of the cyst, stereotactic cyst aspiration, endoscopic cyst fenestration, or shunt placement. Fenestration through either approach is often preferred to upfront shunt placement to avoid shunt-related complications., , 17,18 Craniotomy and endoscopic fenestration allow inspection of the cyst wall, ${ }^{2,12,18}$ coagulation of arachnoidal blood ves-

TABLE 4

Complications following surgery for arachnoid cysts

\begin{tabular}{lc}
\hline \hline \multicolumn{1}{c}{ Complication } & No. of Cases \\
\hline none & 11 \\
weakness & 3 \\
reaccumulation & 5 \\
required shunt placement & 3 \\
CSF leak & 1 \\
seizure & 1 \\
subural hygroma & 1 \\
\hline
\end{tabular}

sels, ${ }^{8}$ and pathological diagnosis confirmation with a tissue biopsy procedure. Fenestration tends to be especially favored over shunting for cyst locations in which vital structures may be injured during shunt placement or following cyst wall collapse.

Advantages of open cyst fenestration via craniotomy include excision of the cyst wall, possible fenestration of multiloculated cysts, and, in certain locations, fenestration of the cyst into neighboring cisterns. ${ }^{10,17}$ Despite the absence of the immediate reexpansion of displaced structures on postoperative imaging, patients report rapid resolution of symptoms such as headache. However, headaches may return in 5 to $25 \%$ of patients because of cyst reaccumulation. ${ }^{10,17,25}$

Upfront CP shunt insertion has been proposed in patients with concomitant or resultant hydrocephalus ${ }^{2,6-8,26}$ (based on reports of high reaccumulation rates following fenestration $^{6}$ ) as well as in those with arachnoid cystrelated ventriculomegaly that fails to resolve after cyst fenestration. Cystoperitoneal shunt placement remains an appealing option given the simplicity of the surgical technique involved and the availability of intraoperative guidance via ultrasonography or neuronavigation, but complications inherent to all CSF drainage systems must be considered. The development of slit-cyst syndrome (a condition similar to slit-ventricle syndrome) is also possible following CP shunt placement and should be considered on neurological deterioration in the patient. Reported rates of shunt revision in this patient population are approximately $30 \% .^{10,25}$

Endoscopic cyst fenestration has gained popularity with excellent reported outcomes. ${ }^{22,23}$ Magnetic resonance imaging- or computed tomography-guided endoscope trajectories are used, access to the cyst is gained via coagulation of the outer cyst membrane, and generous cystocisternostomies are performed. ${ }^{23}$ Neuroendoscopic access has also been used as a complement to open microsurgical fenestration. ${ }^{13}$ As this technique gains exposure, minimally invasive fenestration may become the preferred surgical technique for accessible cysts.

\section{Conclusions}

In summary, recent advances in, and greater access to, neuroimaging have increased the detection of arachnoid cysts. Most cysts are found incidentally and can be managed conservatively with periodic imaging. Symptomatic patients are candidates for surgical intervention via several methods, including CP shunt placement, craniotomy, endoscopic fenestration, and stereotactic aspiration. Recent advances in neurosurgical techniques and neuroendoscopy continue to favor fenestration over shunting as the method of choice for initial cyst decompression.

\section{References}

1. Albuquerque FC, Giannotta SL: Arachnoid cyst rupture producing subdural hygroma and intracranial hypertension: case reports. Neurosurgery 41:951-956, 1997

2. Anderson FM, Segall HD, Caton WL: Use of computerized tomography scanning in supratentorial arachnoid cysts. A report on 20 children and four adults. J Neurosurg 50: 333-338, 1979 
3. Becker T, Wagner M, Hofmann E, Warmuth-Metz M, Nadjmi $\mathrm{M}$ : Do arachnoid cysts grow? A retrospective CT volumetric study. Neuroradiology 33:341-345, 1991

4. Bright R: Serous cysts in the arachnoid, in Rees, Orme, Brown, Green (eds): Diseases of the Brain and Nervous System. Part I. London: Longman Group Ltd, 1831, pp 437-439

5. Caemaert J, Abdullah J, Calliauw L, Carton D, Dhooge C, van Coster R: Endoscopic treatment of suprasellar arachnoid cysts. Acta Neurochir (Wien) 119:68-73, 1992

6. Ciricillo SF, Cogen PH, Harsh GR, Edwards MS: Intracranial arachnoid cysts in children. A comparison of the effects of fenestration and shunting. J Neurosurg 74:230-235, 1991

7. di Rocco C, Caldarelli M, di Trapani G: Infratentorial arachnoid cysts in children. Childs Brain 8:119-133, 1981

8. Galassi E, Tognetti F, Frank F, Fagioli L, Nasi MT, Gaist G: Infratentorial arachnoid cysts. J Neurosurg 63:210-217, 1985

9. Handa J, Okamoto K, Sato M: Arachnoid cyst of the middle cranial fossa: report of bilateral cysts in siblings. Surg Neurol 16:127-130, 1981

10. Harsh GR IV, Edwards MS, Wilson CB: Intracranial arachnoid cysts in children. J Neurosurg 64:835-842, 1986

11. Higashi S, Yamashita J, Yamamoto Y, Izumi K: Hemifacial spasm associated with a cerebellopontine angle arachnoid cyst in a young adult. Surg Neurol 37:289-292, 1992

12. Hoffman HJ, Hendrick EB, Humphreys RP, Armstrong EA: Investigation and management of suprasellar arachnoid cysts. J Neurosurg 57:597-602, 1982

13. Hopf NJ, Perneczky A: Endoscopic neurosurgery and endoscope-assisted microneurosurgery for the treatment of intracranial cysts. Neurosurgery 43:1330-1337, 1998

14. Jamjoom ZA, Okamoto E, Jamjoom AH, al-Hajery O, AbuMelha A: Bilateral arachnoid cysts of the sylvian region in female siblings with glutaric aciduria type I. Report of two cases. J Neurosurg 82:1078-1081, 1995

15. Lemire R, Loeser J, Leech R, Alvord EJ: Normal and Abnormal Development of the Human Nervous System. New York: Harper \& Row, 1975
16. Prabhu VC, Bailes JE: Chronic subdural hematoma complicating arachnoid cyst secondary to soccer-related head injury: case report. Neurosurgery 50: 195-198, 2002.

17. Raffel C, McComb JG: To shunt or to fenestrate: which is the best surgical treatment for arachnoid cysts in pediatric patients? Neurosurgery 23:338-342, 1988

18. Raimondi AJ, Shimoji T, Gutierrez FA: Suprasellar cysts: surgical treatment and results. Childs Brain 7:57-72, 1980

19. Rengachary SS, Watanabe I: Ultrastructure and pathogenesis of intracranial arachnoid cysts. J Neuropathol Exp Neurol 40: 61-83, 1981

20. Rengachary SS, Watanabe I, Brackett CE: Pathogenesis of intracranial arachnoid cysts. Surg Neurol 9:139-144, 1978

21. Santamarta D, Aguas J, Ferrer E: The natural history of arachnoid cysts: endoscopic and cine-mode MRI evidence of a slitvalve mechanism. Minim Invasive Neurosurg 38:133-137, 1995

22. Schroeder HW, Gaab MR: Endoscopic observation of a slitvalve mechanism in a suprasellar prepontine arachnoid cyst: case report. Neurosurgery 40:198-200, 1997

23. Schroeder HW, Gaab MR, Niendorf WR: Neuroendoscopic approach to arachnoid cysts. J Neurosurg 85:293-298, 1996

24. Smith RA, Smith WA: Arachnoid cysts of the middle cranial fossa. Surg Neurol 5:246-252, 1976

25. Stein SC: Intracranial developmental cysts in children: treatment by cystoperitoneal shunting. Neurosurgery 8:647-650, 1981

26. Vaquero J, Carrillo R, Cabezudo JM, Nombela L, Bravo G: Arachnoid cysts of the posterior fossa. Surg Neurol 16:117-121, 1981

Manuscript submitted January 15, 2007.

Accepted January 23, 2007.

Address reprint requests to: George Jallo, M.D., Department of Neurosurgery, The Johns Hopkins University, School of Medicine, Harvey 811, 600 North Wolfe Street, Baltimore, Maryland 21287. email: gjallo1@jhmi.edu. 